\title{
Reflections on the Compatibility, Performance, and Scalability of Parallel Python
}

\author{
Remigius Meier \\ Department of Computer Science \\ ETH Zurich \\ Zürich, Switzerland \\ remi.meier@inf.ethz.ch
}

\author{
Thomas R. Gross \\ Department of Computer Science \\ ETH Zurich \\ Zürich, Switzerland \\ thomas.gross@inf.ethz.ch
}

\begin{abstract}
Today's hardware is increasingly parallel, and to increase performance, applications must be able to use this parallelism. Hence, programming languages must provide the means for parallel execution. The language Python offers a multithreading, shared-memory model for concurrency. However, simultaneous execution of threads, i.e., parallel execution, is not a standard feature of current virtual machines (vM) for Python. Instead, the predominant Python vms depend on a global interpreter lock, which serializes the execution.

In a parallel vM, replicating Python's concurrency semantics is challenging. Today, there are three parallel vMs, which use one of two approaches to address the challenges: JYTHON, IronPython, and PyPy-stm. These vms use two fundamentally different approaches to synchronize parallel execution under Python's concurrency semantics: JyTHON and IRONPYTHON use fine-grained locking, and PyPY-STM uses software transactional memory (STM).

The two approaches result in different performance characteristics and levels of Python compatibility for these vas. In this paper, we report on our experience with the three parallel vms by comparing their compatibility, performance, and scalability. The comparison shows that fine-grained locking can yield better scalability than the STM approach. However, regarding the faithful reproduction of Python's concurrency semantics and the absolute performance, the STM approach currently has the advantage.
\end{abstract}

CCS Concepts - Software and its engineering $\rightarrow$ Parallel programming languages; Runtime environments; Interpreters; Just-in-time compilers.

Keywords parallelism, dynamic language, virtual machine, language runtime

Permission to make digital or hard copies of part or all of this work for personal or classroom use is granted without fee provided that copies are not made or distributed for profit or commercial advantage and that copies bear this notice and the full citation on the first page. Copyrights for thirdparty components of this work must be honored. For all other uses, contact the owner/author(s).

DLS '19, October 20, 2019, Athens, Greece

(c) 2019 Copyright held by the owner/author(s).

ACM ISBN 978-1-4503-6996-1/19/10.

https://doi.org/10.1145/3359619.3359747

\section{ACM Reference Format:}

Remigius Meier and Thomas R. Gross. 2019. Reflections on the Compatibility, Performance, and Scalability of Parallel Python. In Proceedings of the 15th ACM SIGPLAN International Symposium on Dynamic Languages (DLS '19), October 20, 2019, Athens, Greece. ACM, New York, NY, USA, 13 pages. https://doi.org/10.1145/3359619. 3359747

\section{Introduction}

Python is one of the most popular programming languages in use today. The dynamic language finds applications in various domains: scientific and numeric applications, network programming, game development, and many more. These tasks require that a Python program can use modern hardware effectively. Hence, the language must provide means for a program to use the increasing parallelism available in modern hardware.

Python provides a concurrent execution model that is based on multithreading in shared memory. With this model, multiple computations can run on multiple threads. However, contrary to the majority of mainstream languages, executing these threads simultaneously, i.e., in parallel, is not at all a standard feature of current Python implementations. Although, such parallel execution is a requirement for using modern hardware to the fullest extent.

The default choice for a Python implementation is $\mathrm{CPY}-$ THON [1], a virtual machine (vM) for Python. CРyтHON is available on all major platforms and serves as the reference implementation of Python itself. Due to this position, it is by far the most widely used Python implementation. Unfortunately, СРутноN does not support the parallel execution of Python code due to relying on coarse-grained locking with a global interpreter lock (GIL). As a consequence, CРYTHON is not a parallel, but a serial vM.

CPyThon's reliance on the GIL is especially unfortunate for parallel Python, i.e., a Python with parallel execution. Due to CPyThon's dominance as the reference implementation, serialized execution is the default experience for Python developers. Thus, a majority of Python developers cannot exploit the parallelism in modern hardware through multithreading in shared memory - one of the most widely used parallel programming models.

Not relying on a GIL, however, is a difficult task for a parallel vm that aims for compatibility with Python semantics. 
The reason is the particular concurrency semantics that were introduced by the use of the GIL in CPyThon: Due to the GIL's serialization of the execution and the ease with which operations on built-in data types can be made atomic, Python's concurrency semantics now includes unconditional sequential consistency for all program executions and atomic operations on large entities, such as the atomic sorting of a list. This semantics gives significantly stronger guarantees than the prevalent hardware on which the Python vM is running. A parallel Python vM, therefore, must emulate this concurrency semantics using potentially complex and expensive synchronization.

Until today, three alternative Python vMs avoid the GIL in an implementation of parallel Python: JyTHON [6], IRONPython [3], and PyPy-stm [21, 22]. These parallel vms use two fundamentally different approaches to synchronize the parallel execution of Python code and to emulate Python's concurrency semantics: JyTHON and IRONPYTHON use finegrained locking, and PyPy-sTM uses software transactional memory (STM). These two emulation approaches yield vMs with different performance characteristics and differing levels of Python compatibility.

In this paper, we report on our experience with the three parallel vMs. The vMs are compared based on their compatibility, performance, and scalability. This comparison yields insights into the state of parallel Python and the differences between the two approaches for implementing Python's concurrency semantics. With this comparison, we answer the question if and when a parallel vM can provide benefits over the currently prevalent serial vMs for Python. The contributions of this paper are the following:

- A discussion of how fine-grained locking and transactional memory address the challenges for a compatible, parallel Python vM.

- A performance comparison of two serial vMs, CPYTHON and PyPy, and the parallel vMs Jython, IronPyTHON, and PyPy-stm.

- Comparison of fine-grained locking with software transactional memory with regard to performance and scalability of the respective vMs.

\section{The State of the Art of Parallel Python}

To understand the difficulty of the task of implementing a parallel Python vM, we first must discuss Python's concurrent execution model and its concurrency semantics. Since Python is an implementation-defined language, we describe Python's concurrent execution model as it is currently implemented by СРутноn, the reference implementation.

Python allows programs to execute program parts concurrently by using threads. Hence, the concurrency structure of Python's execution model is multithreading with shared memory. A thread is essentially an execution stream of concurrent units, and these concurrent units can interact with each other through shared memory. In Python, the concurrent units are individual bytecode instructions from CPyтноN's bytecode interpreter. Examples of bytecode instructions are control flow instructions, reading or updating object attributes, and arithmetic operations. ${ }^{1}$ We call these instructions PYOPS, short for Python operation.

CPyтноN's model for multithreaded, concurrent execution is that of running one interpreter per Python thread. Each interpreter executes Pyops and thereby creates execution streams of PYOPS. Within these streams, the execution of individual pyops is partially ordered by the in-thread sequential order, i.e., the program order. Hence, an interleaving of PYOP executions from multiple Python threads implements concurrency for multithreaded Python programs.

To synchronize the vM under concurrency, CРутHON uses coarse-grained locking. A vM-wide lock, the Global Interpreter Lock (GIL), protects VM-internal data structures against concurrency bugs. The main purpose of the GIL is to make the reference counting for CPYTHON's memory management thread safe. ${ }^{2}$ The GIL is acquired for the execution of individual PyOPs, i.e., the GIL is only released in-between PyOP executions. As a result, PYOP executions are serialized across concurrently executing Python threads.

\subsection{Python's Concurrency Semantics}

Three alternative Python vMs avoid the GIL and thereby gain the ability of executing PYOPS in parallel: JyTHON, IRONPython, and PyPy-stm. However, avoiding the GIL is not a simple feat. The serialized execution, due to the GIL, introduced a particular semantics to the language Python, a semantics that must be reproduced and guaranteed also in the presence of parallel execution.

The serialization of PyOps guarantees that a total order on the execution of Pyops exists. Hence, Python programs execute under sequential consistency (sc) [15]. Additionally, the GIL makes each PYOP execute in a critical section under mutual exclusion. Due to mutual exclusion, ensuring atomic execution for select Pyops is trivial, which led to the introduction of atomic operations (AO) in the language Python.

In particular, PYOPs that operate on built-in data types are guaranteed atomic execution regardless of the size of the data structure involved. Examples of atomic operations are: ${ }^{3}$

- 11 . extend ( 12 ), which appends all elements of the argument list to the target list;

- 11. sort ( ), which sorts a list in place;

- $l 1[i: j]=12$, which overwrites a part of a list with another list's contents.

\footnotetext{
${ }^{1}$ Complete list: https://docs.python.org/2/library/dis.html

${ }^{2}$ Discussed in https://wiki.python.org/moin/GloballnterpreterLock

${ }^{3} 11$ and $l 2$ are lists of built-in numeric types, $i$ and $j$ are integer values. For the complete list, see https://docs.python.org/2/faq/library.html\#whatkinds-of-global-value-mutation-are-thread-safe
} 
A list can be sorted atomically even if the list has many elements and sorting takes a significant amount of time. Atomic operations do not require additional synchronization from the programmer. The atomicity is ensured by synchronization within the Python VM itself.

A compatible, parallel Python vM must provide a parallel execution model that replicates both, sc and Ao. However, what makes this task difficult is that these guarantees are not supported natively by today's prevalent host hardware on which the vM is running. The x86 architecture, for example, does not maintain sc. And for atomic operations, even the recent introduction of Transactional Synchronization Extensions in Intel processors is limited by the size of the on-processor cache, which means that large-scale atomic operations such as list.sort ( ) are out of reach. Hence, any semantic guarantees that go beyond what is offered by the host must be emulated with additional synchronization mechanisms.

The aforementioned parallel Python vMs differ in the way they approach the emulation of Python's concurrency semantics: JYTHON and IRONPYTHON chose the route of making the locking granularity finer. Instead of coarse-grained locking with a single, global lock, locks are placed on individual objects to synchronize concurrent accesses. Thereby, parallel execution becomes possible whenever the sets of locks acquired by two threads are disjoint. In contrast, PyPy-sTM uses software transactional memory to speculatively execute threads in parallel, and then depends on transactional semantics to resolve conflicting accesses.

\subsection{Fine-Grained Locking}

To support parallel execution of Python code, parts of the Python vm itself must execute in parallel, and these parts must, thus, be synchronized correctly. With the model of an interpreter per Python thread, interpreters must synchronize their concurrent accesses to shared memory. With a GIL, interpreters take turns in executing and accessing shared memory. Fine-grained locking, instead, makes the locking granularity finer and places locks on individual objects and data structures. Thereby, multiple interpreters can lock and work on different objects simultaneously.

However, correct synchronization does not merely mean blocking concurrent accesses to the same object. Python's sc additionally requires that accesses must appear to happen in a total order that is consistent with the program order. For example, reading an attribute of a Python object must be synchronized and ordered globally with writing to an attribute in a different thread. And all threads must observe these operations in the exact same order.

JYTHON, as a representative of a vM implemented with fine-grained locking, achieves sc for attribute accesses by using Java's ConcurrentHashMap ${ }^{4}$ to store object attributes

\footnotetext{
${ }^{4}$ https://docs.oracle.com/javase/8/docs/api/java/util/concurrent/ ConcurrentHashMap.html
}

and their values. Each attribute-value pair of an object is a key-value pair in the hash map that belongs to this object. Sequentially consistent behavior for updating and retrieving individual keys is maintained by Java's ConcurrentHashMap implementation.

JythoN uses a ConcurrentHashMap in several places to make operations on built-in data types follow sc, e.g., in the implementations of set and dict. And in other places, data types are made into monitors [14] using Java's synchronized keyword, e.g., in the implementation of list.

Using a monitor to implement sc for list also makes listoperations atomic through mutual exclusion on method calls. This approach works well for the single-object atomic operations listed in Section 2.1. However, to ensure atomicity for operations involving multiple objects, multiple locks must be acquired. Hence, making list into a monitor is not sufficient to implement all documented Aos.

An example of an AO where one monitor is not sufficient is 11 . extend ( 12 ). Both lists, $l 1$ and 12 , must be locked to ascertain mutual exclusion with respect to a concurrent (reverse) operation 12. extend ( 11 ). And if multiple locks are involved, the ordering of lock acquisition becomes significant. If one thread attempts to lock first 11 and then 12 , and another thread attempts to lock 12 and then 11 , both may succeed to lock their first list but will then deadlock when attempting to lock their second list. Hence, a more sophisticated locking scheme is required to avoid deadlocks in multi-object Aos.

As it happens, Jython does not implement the required locking scheme, and, to avoid deadlocks, does not guarantee atomicity for 11 . extend ( 12 ). JYTHON, thus, is not a fully compatible implementation of Python. The way in which JyTHON deals with this issue is evidence for the challenging nature of the fine-grained locking approach to implementing a parallel execution model with Python semantics.

IRONPYTHON improves upon JyTHON's emulation of AOS with the help of an 0rderedLocker. This OrderedLocker acquires the locks of the two involved objects in the order defined by their identity hash codes (or an assigned identity, if the hash codes are the same). Thereby, deadlocks due to lock acquisition order are prevented, and even multi-object Aos execute under mutual exclusion.

As a consequence, IronPython's emulation of Python's concurrency semantics is more complete than JyThoN's. A remaining incompatibility in IRONPYTHON's implementation of multi-object AOs is $l 1[i: j]=12$. In particular, the case of $l 1[:]=12$, where the entire list $l 1$ is replaced with the contents of 12, IRONPyTHON does not fully achieve atomicity. Instead, if a concurrent $l 1[:]=13$ is executing, $l 1$ may end up containing the concatenation of 12 and 13 . The concurrent, atomic executions of $11[:]=12 \mid 13$, however, should make 11 to be either 12 or 13 , not a combination thereof. 
We reported this issue to the project ${ }^{5}$ and the findings were confirmed. At the time of writing, the incompatibility was not yet resolved. Since IronPython correctly emulates other multi-object AOs, we consider this issue as additional evidence for the difficulty in correctly emulating Python's concurrency semantics with fine-grained locking.

For the performance of a Python vM using the fine-grained locking approach, the effective optimization of locking operations is an important factor. JYTHON depends on the synchronization mechanisms offered by Java and its vM: an optimized ConcurrentHashMap with sc guarantees, and synchronized methods for implementing AO with monitors. On modern Java vMs, JyTHON benefits from a set of sophisticated optimizations for lowering the overhead of these synchronization mechanisms [18, 33]. Without such optimizations, or when optimizations fail to apply, the performance cost of fine-grained locking can be significantly higher [16]. The same applies to IronPython, which benefits from the optimizations of the underlying Common Language Runtime.

Still, even though the vMs underlying JyTHON and IRONPython provide powerful optimizations, the described approach to implement AO and SC introduces inefficiencies. Using a hash map for implementing object attributes means that each attribute access involves a hash map lookup. For such a frequent operation, this indirection is expensive. Additionally, data types that are made into monitors now acquire and release a lock around each operation. While optimizations may successfully remove these locking operations on objects that are proven to not escape a thread, in other cases, the proof fails and locking operations must be present to ensure correctness.

To summarize, fine-grained locking can deliver a parallel Python vM, but the approach requires carefully designed locking schemes to implement Python's concurrency semantics in its entirety. A missing lock can lead to incorrect behavior, and thoughtless locking can lead to deadlocks. Even Jython, first released in 2001, and IronPython, first released in 2006, do not implement the complete semantics and still exhibit incorrect behavior. We look at the performance of the finegrained locking approach in Section 3.

\subsection{Software Transactional Memory}

Using a software-based transactional memory (STM), PyPYSTM approaches the emulation of Python's concurrency semantics in a different way. STM supports the atomic, speculative execution of a group of operations in a transaction. Instead of synchronizing accesses on individual objects to achieve SC and AO, code can be wrapped in a transaction and the STM system guarantees a serializable atomic execution.

Serializability guarantees that a total order (the serialization order) on the execution of transactions exists. Or in

\footnotetext{
${ }^{5}$ https://github.com/IronLanguages/ironpython2/issues/621
}

other words, the outcome of a (parallel) schedule of transactions is equivalent to the outcome of some serial schedule of the same transactions.

With atomic execution and the serializability of transaction schedules, emulating AO and sc becomes straightforward: PyPy-sTM ensures that each AO is enclosed in a transaction, thereby letting the AO inherit the transaction's atomicity. For SC, PyPY-STM executes each PYOP in a transaction. Due to the transaction schedule serializability, PYOPS appear to execute in a total order and therefore under sc.

Parallel execution on multiple threads is accomplished by speculatively running several transactions in parallel. The STM system ensures the serializability by aborting and undoing transactions that would otherwise produce a nonserializable result. In other words, if a transaction cannot complete in a way that fulfills serializability, the transaction is reverted and retried. If enough transactions can successfully execute in parallel without such conflicts, multithreading parallelization can pay off by reducing the overall execution time of a program.

In contrast to fine-grained locking, the use of STM makes the complete emulation of Python's concurrency semantics effortless, since the GIL-induced semantics map directly to transactional semantics. However, reaching high performance with an STM system is challenging. For the capabilities of reverting and retrying transactions, as well as for detecting conflicts, the STM system must maintain undo logs and employ read and write barriers. When values are overwritten in a transaction, enough information must be maintained to restore the overwritten values. And read and write barriers are required to detect if a race condition prevents the successful commit of the transaction in the serialization order. Since these mechanisms are executed frequently, significant overhead is introduced.

One approach to lower the overhead of STM is to amortize the work done in a transaction by making the transaction longer. A major part of the bookkeeping of the STM system needs to be done only once per transaction per object. Hence, if more actual work is completed per transaction, the overhead of bookkeeping relative to the completed work becomes lower. However, increasing the length of a transaction also increases the probability that a conflict with another transaction is encountered. Additionally, the longer a transaction, the more work is lost if the transaction must abort. Hence, increasing the length of a transaction trades off the overhead against the risk of an expensive transaction abort. Both can be detrimental to performance, and PyPy-sTM applies an adaptive heuristic to strike a balance between the two.

To summarize, an approach based on transactional memory can deliver a parallel vM with the complete concurrency semantics of Python. The emulation of the semantics is straightforward, but the approach depends on a carefully tuned STM system to reach high performance. Bookkeeping and conflict detection introduce overhead throughout the 
VM, and wrong decisions by the sTM system may lead to performance degradation. For the Python vM, conceptually, the sтM-based approach is less complex than fine-grained locking, but the performance overhead may be higher than the highly-optimized locks and concurrent data structures used in JyTHON and IRONPYTHON.

\section{Evaluation}

In the previous section, we presented two approaches to emulating Python's concurrency semantics in a parallel vM. The first, fine-grained locking, finds use in JyTHON and IRONPython, two parallel Python vms that depend heavily on the vms of their implementation languages, Java and C\#. The Java VM (JVM) and the Common Language Runtime (CLR) optimize locking operations, provide synchronized data structures, and even provide just-in-time (JIT) compilation as a VM service. JythoN and IrONPython both use the underlying JIT compiler to compile the code of Python applications for better performance.

The second approach, software transactional memory (STM), is used in PyPy-stm. PyPy-stm is based on PyPy, a highperformance Python vMs that leverages a meta-tracing JiT compiler. Like CPython, PyPy uses a GIL. Because PyPy-STM uses a modified version of the same JIT compiler as PyPy, the two vMs share many performance characteristics.

In this section, we report the performance levels of these three parallel Python vMs relative to the performance levels of the two serial vMs, CPyтноN and PyPy.

\subsection{Python vMs}

In this evaluation, we run a set of benchmark programs on several Python vMs and measure the programs' execution times. We choose to include two baseline vMs that do not support parallel execution, but that serve as reference points because of either their prevalent usage or their high performance. The two serial vMs are:

CPython [1] Python's reference implementation, a serial VM without a JiT. Version: 2.7.12

PyPy [7] A serial Python vM with a tracing JIT compiler. The Jit compiles Python code down to native machine code for the host CPU. Version: 5.7.0

The parallel Python vas under evaluation are:

Jython [6] A parallel Python vм built on top of the JVM, uses the fine-grained locking approach, compiles Python code down to Java bytecode and thereby benefits from the JVM's JiT compiler. Version: 2.7.0

IronPython [3] A parallel Python vm built on top of the CLR, uses the fine-grained locking approach, compiles Python code using the Dynamic Language Runtime (DLR [2]), which leverages the CLR's JIT compiler. Version 2.7.9 on .NET Core 2.2. ${ }^{6}$

\footnotetext{
${ }^{6}$ The recommended way to run IronPython on Linux is using Mono as the CLR, but we found that .NET Core delivers significantly better performance.
}

Table 1. The parameters used for the benchmarks with normal and increased workloads.

\begin{tabular}{l|r|r} 
Benchmarks & Normal & Increased $\star$ \\
\hline fannkuch & 10 & 11 \\
nucleotide & (default) & (not available) \\
mandelbrot & 128768768 & 128 4096 2048 \\
mersenne & 2000 & 3000 \\
nqueens & 9 & 10 \\
parsible & (default) & (not available) \\
perlin-ex & 16 & 300 \\
raytrace & 256512 & 51204096 \\
regex-dna & small & (default) \\
richards & 30 & 10000
\end{tabular}

PyPy-stm [21, 22] A parallel Python vm, uses the sтм approach and a tracing JIT compiler. The JIT compiler is an STM-enabled version of PyPy's JiT. Version: 5.7.0

PyPy's JiT compiler requires warming up to reach a steady state in performance, and for short execution times below 1 second, PyPy's performance is often lower than CPутноN's. Hence, PyPy's benefits take effect with long-running applications. Here, we only measure steady-state performance, i.e., the reported execution times always represent the performance of a warmed-up JiT compiler.

JyTHON is the oldest representative for the fine-grained locking approach to a parallel Python vM. Its fields of application are more limited than СРүтноN's due to its reliance on the JVM. IronPython follows in the footsteps of JyTHON and also uses the fine-grained locking approach to a parallel Python vM. The architecture of IRONPyTHON is different due to its use of the DLR, and, in contrast to JYTHON, IRONPYTHON aims for full compatibility with Python even in the case of multi-object AOs.

PyPy-stm is built with the Parallel RPython framework [2022], an evolution of the RPython [29] framework used to develop PyPy. Hence, PyPy-STM shares code and many performance characteristics with PyPy. The major differences are the capability of speculative parallel execution using STM and a different garbage collection implementation. PyPY-STM faithfully reproduces Python's concurrency semantics.

\subsection{Benchmarks}

To understand the performance characteristics of the three parallel vMs, we use a collection of multithreaded Python programs [8] and measure their execution times. We collected these programs from several sources ${ }^{7}$ and, since Python programs that use multithreading for performance are still rare, manually parallelized a selection of them for our evaluation. The benchmarks do not make use of Aos that involve multiple objects, which would require extra synchronization for JYTHON (and potentially IRONPYTHON).

\footnotetext{
${ }^{7}$ The origin of a benchmark is noted as a comment in the source file.
} 
Table 2. Benchmark characterization showing lines of Python code (LoC), the performed task, and the dominant computation.

\begin{tabular}{|c|c|c|c|}
\hline Benchmarks & LoC & Task & Dominant computation \\
\hline fannkuch & 266 & Fannkuch game & list manipulations \\
\hline nucleotide & 222 & $\begin{array}{l}\text { DNA } \\
\text { frequencies }\end{array}$ & $\begin{array}{l}\text { string slicing, sorting, } \\
\text { searching }\end{array}$ \\
\hline mandelbrot & 239 & Mandelbrot set & $\begin{array}{l}\text { complex number } \\
\text { calculations }\end{array}$ \\
\hline mersenne & 236 & $\begin{array}{l}\text { Mersenne } \\
\text { primes }\end{array}$ & big integer calculations \\
\hline nqueens & 217 & $\begin{array}{l}\text { N-Queens } \\
\text { problem }\end{array}$ & list/set iteration, generators \\
\hline parsible & 479 & Log parsing & $\begin{array}{l}\text { regular expression } \\
\text { matching, input/output }\end{array}$ \\
\hline perlin-ex & 269 & Perlin noise & integer operations \\
\hline raytrace & 302 & Ray tracing & $\begin{array}{l}\text { object manipulations, float } \\
\text { calculations }\end{array}$ \\
\hline regex-dna & 223 & $\begin{array}{l}\text { DNA gene } \\
\text { search }\end{array}$ & $\begin{array}{l}\text { regular expression } \\
\text { matching, input/output }\end{array}$ \\
\hline richards & 497 & $\begin{array}{l}\text { OS kernel } \\
\text { simulation }\end{array}$ & object manipulations \\
\hline
\end{tabular}

The benchmarks are pure Python programs and therefore run on all vMs. A benchmark can be configured to distribute its workload on any number of threads. Hence, on a parallel VM, we expect a reduction in the benchmark's execution time with an increasing number of available threads. In the Normal column of Table 1, we list the additional parameters that are passed to each benchmark program. The Increased $\star$ column is relevant for Section 3.6.

The benchmarks perform various tasks, as shown in Table 2 . In all benchmarks, the concurrency pattern follows the fork-join model, and the computations on concurrent threads are largely independent. Even though each program is small, they exercise various aspects of a vM, and we argue that these programs give insights into the class of applications that can profit from the same, prevalent concurrency pattern.

\subsection{Host Environment}

Initial measurements are done with up to 8 Python threads. The limit of 8 threads is a deliberate choice to represent the prevalent host environment of Python vMs. Python programs often execute on commodity desktop computers that include between two and eight processor cores. It is in such lowcore environments that parallelization must pay off for a parallel Python vM to be useful. Nevertheless, we evaluate the situation beyond the limit of 8 threads in Section 3.6.1.

Our evaluation system, however, offers plenty of resources: The system has 512 GB of memory and two Intel Xeon E52699v4 CPUs (release date: 2016), each with 22 cores. But for the aforementioned reasons, we limit the benchmark programs in the numbers of threads they can use, and we limit all benchmarking to a single CPU with 22 cores and 256 GB of memory to minimize the impact of Non-Uniform-MemoryAccess (NUMA) effects. The restriction was enforced with numact ${ }^{8}$ by restricting execution and memory allocation to node 1 . Thread scheduling within the node was left to the operating system. ${ }^{9}$

\subsection{Methodology}

The vMs are tested in their default configurations and the vM startup time is excluded in our measurements. Before measuring a benchmark's execution time, we first run a manually configured, benchmark-specific number of warm-up iterations. The number of warm-up iterations was determined by manually inspecting the measurements to see when a benchmark reached a steady state. Note that the steady state does not always imply peak performance [9, 17]. However, we argue that the steady state is representative of the experienced performance and therefore relevant for a vM comparison.

Unless noted otherwise, the reported time is the mean of 18 measured benchmark iterations. These 18 iterations result from 6 fresh VM instances, each doing 3 in-vM benchmark iterations. The figures include the standard deviation as error bars. For comparing the speedup of two configurations, we report the mean over all possible pairings of the $2 \times 18$ measured execution times.

Before every benchmark iteration, a call to gc . collect ${ }^{10}$ allows the vM to free up memory. Hence, garbage collection cycles should influence the measurements only if they are systematically induced within a benchmark iteration. Similarly, we disable future just-in-time compilation for $\mathrm{PyPy}$ and PyPy-stM after warm-up. The underlying vMs of IRONPython and Jython, i.e., the CLR and the JVM, do not support this functionality. Consequently, the measurements can be influenced by systematic garbage collection and JIT compilation. Still, the validity of the relative performance results is confirmed by the low standard deviations in the results.

\subsection{Performance Classification}

To obtain an overview of the situation of parallel Python, we look at the performance levels of the Python vM representatives and make a performance classification. First, we compare single-threading, i.e., sequential, performance of the vMs on the set of benchmarks. This comparison allows for a classification relative to the two serial baseline vMs, CPython and PyPy.

Figure 1 shows the relative speedup of the vMs on all benchmarks with CРутноN as the baseline. The height of the bar is the speedup, meaning how much faster a benchmark iteration completed on a VM as compared to CPyTHON. The legend additionally shows for each vm the geometric mean of speedups over all benchmarks.

\footnotetext{
$\overline{8}$ https://linux.die.net/man/8/numactl

${ }^{9}$ Linux 4.4.0-65-generic Ubuntu x86_64

${ }^{10}$ See https://docs.python.org/2/library/gc.html (all evaluated vms implement this functionality)
} 


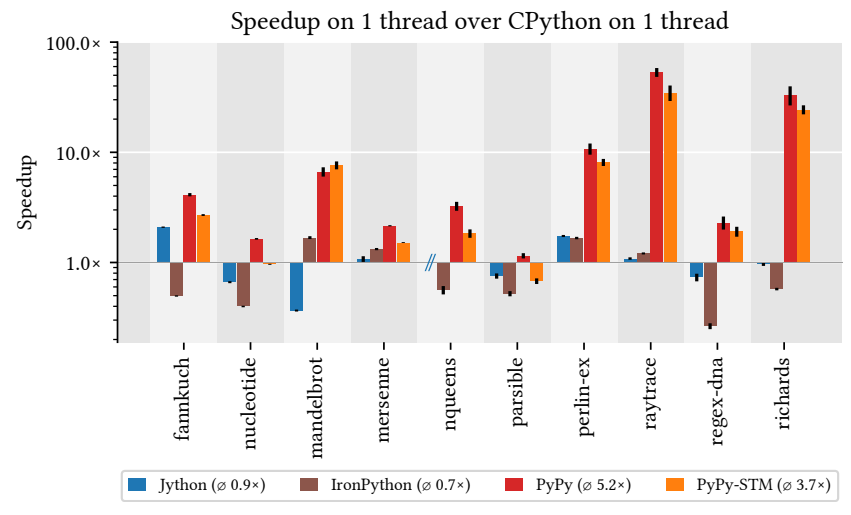

Figure 1. Speedup of single-threaded execution relative to CPyтноn (higher is better). Missing values are indicated with //. The geometric mean speedup for each VM is shown in the legend.

JYTHON runs out of memory on nqueens, therefore no result is reported for this benchmark. Apart from that, JyTHON performs better than СРутноN in around half of the benchmarks, and worse in the other half. In both cases, the performance differences can be rather significant. The speedups of JyTHON are between $2 \times$ faster and $2.7 \times$ slower. The geometric mean of JyTHON's speedups is $0.9 \times$, and JythoN's sequential performance is, therefore, roughly on par with CPyтноn's (albeit with a large variance).

IRONPYTHON performs similarly to JyTHON. In 4 out of 10 benchmarks, IronPython is up to $1.7 \times$ faster, and in 6 benchmarks up to $2.9 \times$ slower than CPyThon. With a geometric mean speedup of $0.7 \times$, IRONPyTHON is slower than CPyтноn, but still manages to outperform CPyтноN significantly in several cases.

PyPy displays the fastest execution among these vMs (mean speedup of $5.2 \times$ ), and PyPy-STM often takes $2^{\text {nd }}$ place (mean speedup of $3.7 \times$ ). A notable behavior is exhibited by $\mathrm{PyPy}$ on parsible. PyPy with its JIT compiler is barely faster than CPyтнon on this benchmark. parsible is also the one benchmark where JyTHON outperforms PyPy-sTM. The reason for this behavior is that parsible heavily depends on regular expressions. $98 \%$ of the CPU time is spent in matching regular expressions. Hence, this benchmark is more a test of the regular expression engine used by the Python vM than it is a test of Python performance. Consequently, the JIT compilers of PyPy and PyPy-stm can only speed up the remaining 2\% of the time that is spent in Python code, which does not have a significant effect on the overall execution time.

This comparison of the single-threading performance lets us classify the vMs in roughly two levels of performance: medium and high. On average, Jython and CPyThon exhibit approximately the same execution times. The mean speedup of JyTHON with $0.9 \times$ is close enough to 1 to consider both to be in the same performance class, i.e, as mediumperformance vMs. IRONPYTHON, while slower with its mean speedup of $0.7 \times$, still often outperforms CPyтhon. Hence, we also classify IronPython as a medium-performance vM. PyPy and PyPy-sTM are classified as high-performance vMs. These vMs both show more than $3 \times$ mean speedup over CPyтноN and are rarely slower than the other vMs.

This classification puts JyTHON and IRONPyтноN together with CPyтhon. However, Jython and IronPython are capable of parallel execution and can therefore take advantage of the parallelism in modern hardware. Thus, a classification based on the multithreaded execution of benchmarks should turn out differently.

\subsubsection{Parallel Performance Levels}

For each benchmark, Figure 2 shows the execution times on the vMs with 1 to 8 threads available. nqueens is still missing for JyThON. This figure not only shows raw numbers, it also allows for insights about the behavior of the vMs: First, CPyтHON does not display medium performance on multiple threads. Instead, the performance of CPyTHON actually degrades significantly with each additional thread. PyPy similarly slows down in many cases, albeit less extremely. And second, Jython, IronPython, and PyPy-stm exhibit faster execution with more threads available. As a result, the performance classification changes.

Figure 3 again shows the relative speedup of the vMs compared to CРYтHON's single-threading performance, but this time with 8 threads available. On CPyтHon, a benchmark takes, on average, $2.5 \times$ more time with 8 threads than with just 1 thread (mean speedup of $0.4 \times$ ). PyPy's 8-threaded execution time is still $3.3 \times$ faster than the single-threaded execution on CPyтноN, but PyPy's speedup in single-threaded execution was $5.2 \times$ (Figure 1 ). Thus, PyPy slowed down by a factor of $1.6 \times$ when using 8 instead of 1 threads.

With 8 threads, JyThoN is now $3.4 \times$ faster than CPyтноN, and thus roughly on par with PyPy. IronPython also manages to reach a significant speedup of $2.4 \times$ over CPyтHON and is now only slightly slower than Jython and PyPy. And PyPy-sTM is now $13 \times$ faster (up from $3.7 \times$ on a single thread).

Due to CPyтhon's significant slowdown, it can only be considered to reach a new performance level of low. On the other end of the spectrum, PyPy-STm marks the new highperformance level. And between the two extremes, Jython, IronPython, and PyPy achieve medium performance.

The underlying reason for slowing down with multiple threads on PyPy and CPyTHon is the GIL. If the GIL serialized the execution without additional overhead, the lines in Figure 2 would be flat. However, with multiple active threads, the contention on the GIL increases. In general, if a lock cannot be acquired, the acquiring thread goes to sleep and must be woken up when the lock is released. Waking up a thread takes time, and the more contention on a lock, the more 

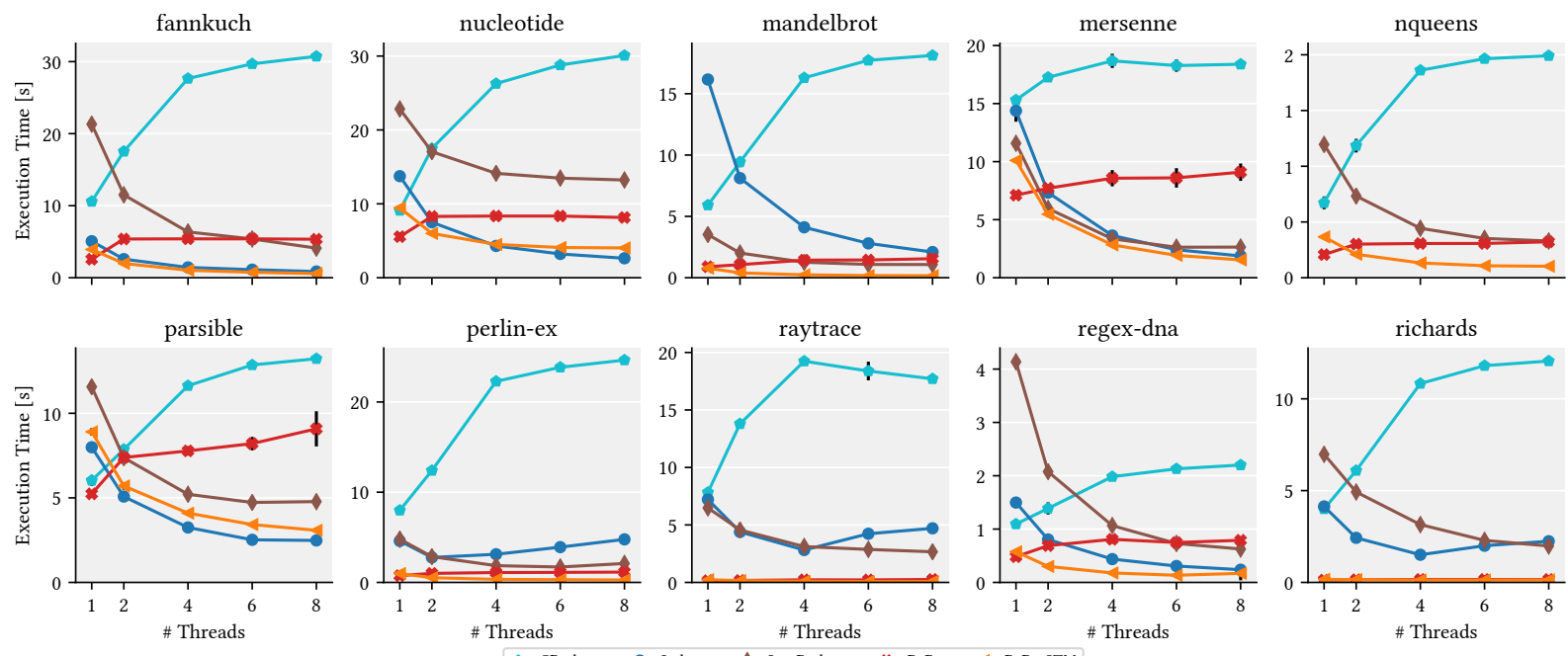

Figure 2. Absolute execution times of benchmark iterations. The number of available threads varies between 1 and 8 .

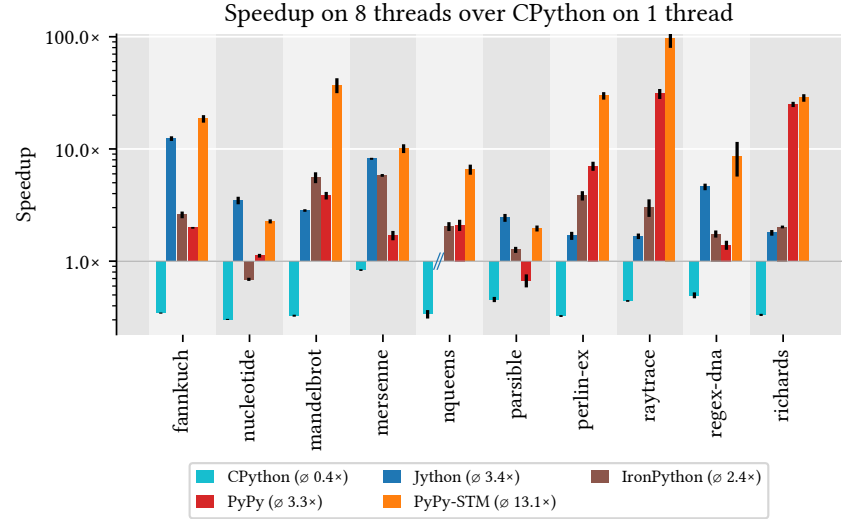

Figure 3. Speedup of 8 -threaded execution relative to the single-threaded execution on CPYтно (a higher bar is better). Missing values are indicated with //. The geometric mean speedup of each VM is shown in the legend.

frequently threads must be woken up. Additionally, lock implementations may wake up multiple threads at the same time; and of those threads, only one can actually acquire the lock; the other threads must go to sleep again. Hence, with an increasing number of threads, the GIL-acquisition takes up increasing amounts of CPU time. And as a consequence, both PyPy and CPyтнon drop one class in performance.

Additionally, the slowdown on multiple threads on GILbased vms has the following consequence: In a scenario where background threads are used to increase the responsiveness of an application, the application's overall performance degrades. For example, a Python application may start a long-running task on a background thread while the main thread handles the user interface. What Figure 2 shows is that the program execution can slow down by up to a factor of $2 \times$ with two threads, and greater than $2 \times$ with higher numbers of threads. In such scenarios, the performance advantage of a parallel vM not only comes from performance gains through parallel execution, but also from simply not slowing down with multiple active threads - a feat achieved by all parallel vms evaluated here.

In contrast to GIL-based vMs, JyThon, IronPyThon, and PyPy-sтm profit from increasing numbers of threads and stay in their performance class. JYTHON, although a performance class below PyPy-STM, often approaches (and sometimes overtakes) the performance of PyPy-sTM.

However, the picture for the parallel vMs is not yet clear. In many instances, the absolute execution times on parallel vms are low and the scalability thereby limited. If the total execution time of a benchmark is too low, distributing the workload onto multiple threads often gives no benefits due to the additional overhead of thread management. Hence, to reveal the entire picture of parallel Python performance, we increase the workload for the following benchmarks (marked with $\star$ ): fannkuch $\star$, mandelbrot $\star$, mersenne $\star$, nqueens $\star$, perlin-ex $\star$, raytrace $\star$, regex-dna $\star$, and richards $\star$.

\subsection{Comparison with Increased Workloads}

The new parameters for the benchmarks are shown in Table 1 in the Increased $\star$ column, and the effect of the increased workload is shown in Table 3. Single-threaded execution times increase by factors between $4 \times$ and $213 \times$. Unfortunately, the increased workloads make the evaluation of $\mathrm{CPY}-$ THON impractical. CPYTHON often exceeds a time limit of 60 min while running a benchmark, which makes collecting a reasonable number of measurements impractical. In the following evaluation, СРутноN is therefore missing, and PyPy's single-threading performance is the new baseline. 
Table 3. Changes in the benchmarks' single-threaded execution times with increased workloads (in seconds). Missing values are due to reaching a 60 min time limit.

\begin{tabular}{|c|c|c|c|c|}
\hline Benchmarks & JYTHON & $\begin{array}{c}\text { IRONPY- } \\
\text { THON }\end{array}$ & PyPy & PyPy-stm \\
\hline fannkuch $\star$ & $5.1 \rightarrow 60.1$ & $21.3 \rightarrow 261.9$ & $2.6 \rightarrow 30.5$ & $3.9 \rightarrow 46.5$ \\
\hline mandelbrot $\star$ & $16.2 \rightarrow 223.6$ & $3.5 \rightarrow 47.8$ & $0.9 \rightarrow 10.5$ & $0.8 \rightarrow 9.8$ \\
\hline mersenne $\star$ & $14.4 \rightarrow 62.1$ & $11.6 \rightarrow 47.5$ & $7.1 \rightarrow 28.7$ & $10.1 \rightarrow 41.5$ \\
\hline nqueens $\star$ & - & $1.2 \rightarrow 11.4$ & $0.2 \rightarrow 1.6$ & $0.4 \rightarrow 2.9$ \\
\hline perlin-ex $\star$ & $4.6 \rightarrow 84.0$ & $4.8 \rightarrow 89.7$ & $0.8 \rightarrow 12.4$ & $1.0 \rightarrow 16.1$ \\
\hline raytrace $\star$ & $7.2 \rightarrow$ & $6.5 \rightarrow$ & $0.1 \rightarrow 13.0$ & $0.2 \rightarrow 18.8$ \\
\hline regex-dna $\star$ & $1.5 \rightarrow 76.2$ & $4.1 \rightarrow 229.9$ & $0.5 \rightarrow 10.1$ & $0.6 \rightarrow 11.5$ \\
\hline richards $\star$ & $4.1 \rightarrow \quad-$ & $7.0 \rightarrow$ & $0.1 \rightarrow 26.6$ & $0.2 \rightarrow 34.7$ \\
\hline
\end{tabular}
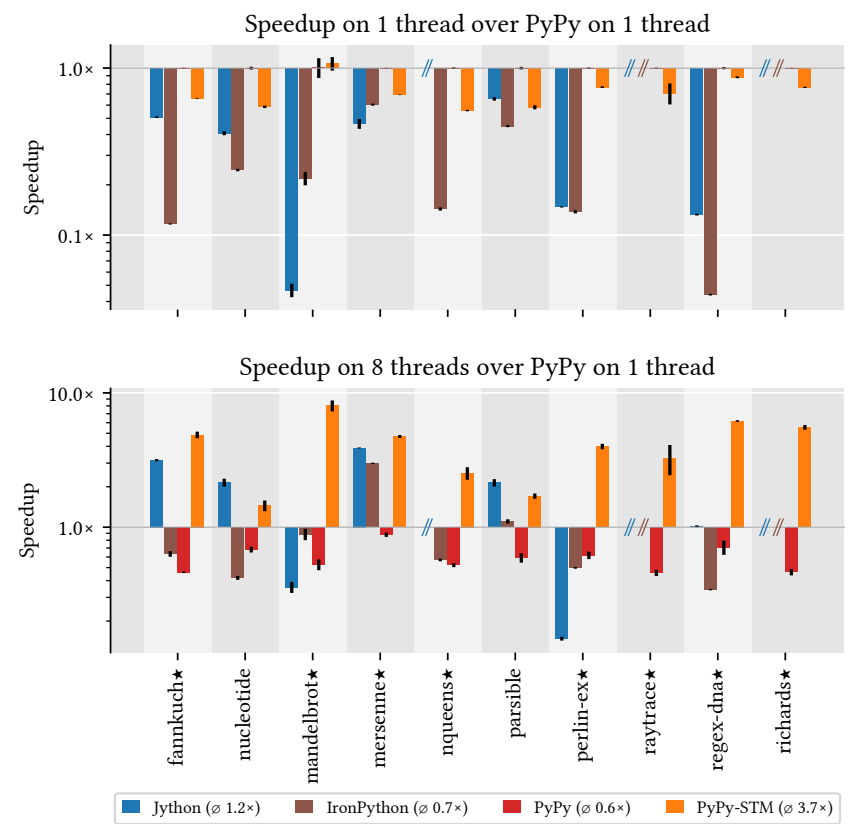

Figure 4. Speedup of single- and 8-threaded execution relative to PyPy's single-threaded execution (higher bars are better). The legend shows the geometric mean for each vM over all benchmarks on 8 threads. Missing values are indicated with $/ /$.

JYTHON manages to run 5 out of the 8 benchmarks with increased workloads. In nqueens $\star$, raytrace $\star$, and richards $\star$, however, JyTHon exceeds the time limit as well. IRONPyTHON manages to run more of the benchmarks than JyTHON, but also exceeds the time limit on raytrace $\star$ and richards $\star$.

Figure 4 shows the speedups for single-threaded and for 8-threaded executions relative to the PyPy baseline. The upper half shows single-threaded execution. With the larger workloads, the performance gap between PyPy and JyTHON and IronPython increases. By increasing the workload, the slowdown of Jython relative to PyPy changes from $2.5 \times$ to $2.9 \times .{ }^{11}$ For IronPython, the change is from $3.4 \times$ to $4.1 \times$.

\footnotetext{
${ }^{11}$ Average over benchmarks that JYTHON runs with the increased workload.
}

PyPy-stm also exhibits lower performance than PyPy due to the synchronization overhead of the sтм. ${ }^{12}$ On average, PyPy-stm's sequential performance is $30 \%$ lower than PyPy (on both, light and heavy workloads) due to the extra synchronization throughout the vM. Still, PyPy-STM remains significantly faster than JYTHON in all but one benchmark. parsible is still an exception where JyTHON leads in the regular expression matching.

IRONPYTHON remains slightly slower than Jython, but outperforms JутноN in mandelbrot $\star$ and mersenne $\star$. Additionally, IronPython manages to run the nqueens $\star$ benchmark, where JyTHon runs out of memory. Hence, while JyTHON has a performance advantage over IRONPYTHON, the result is not clear-cut.

In the lower half of Figure 4, the speedups with 8 threads available are shown. Due to PyPy's reliance on the GIL, we observe that PyPy slows down by an average of $1.7 \times$ (a speedup of $0.6 \times$ ) compared to its single-threading performance. In contrast, JyTHON achieves a mean speedup of $1.2 \times$ relative to a single-threaded PyPy execution. Note, however, that these numbers include mandelbrot $\star$, perlin-ex $\star$, and regex$d n a \star$, where JythoN is particularly slow; and they do not consider nqueens $\star$, raytrace $\star$, and richards $\star$, where JyTHON reaches the $60 \mathrm{~min}$ timeout. This number should therefore be regarded with appropriate caution.

IronPython, even with multiple threads, cannot compete with PyPy's single-threaded performance. The mean speedup of IronPython over PyPy is $0.7 \times$. Only on mersenne and parsible does IronPythoN's scalability improve the execution time to a level that beats PyPy. Compared to Jython,

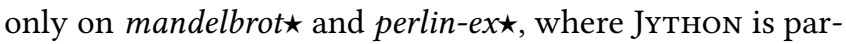
ticularly slow, does IronPython exhibit faster execution times. Generally, IronPython cannot compensate for its lower single-threading performance compared to JyTHON.

PyPy-stm achieves a mean speedup of $3.7 \times$ relative to the baseline. Compared with Jython, PyPy-stm's performance on 8 threads is on the same level for benchmarks that run well on Jython. That is, on benchmarks where JythoN is not particularly slow, the vMs reach a similar level of performance. This high sensitivity to the benchmark makes it difficult to draw a conclusion regarding the relative performance of PyPy-STM and Jython. It is not clear if Jython's extreme cases are performance bugs, or if they are inherent

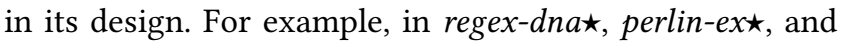
mandelbrot $\star$, JyTHON and IRONPyTHON exhibit low performance, the same is true for the benchmarks where they both reach the time limit, raytrace $\star$ and richards $\star$. These results suggest that JYTHON and IRONPYTHON share a property that makes them perform worse on this kind of programs compared to PyPy-STM. However, it is unclear if this property

\footnotetext{
${ }^{12}$ The exception of mandelbrot $\star$ is due to $2^{\text {nd }}$ order effects, and the special characteristics of this benchmark have been noted before.
} 
is the fine-grained locking approach or, e.g., the layered VM architecture with a managed VM underneath (JVM or CLR).

\subsubsection{Scalability Comparison}

In the above evaluation, we found that JyтноN is generally slower than PyPy-sтм in single-threaded execution. But with 8 threads, JyTHON's performance can approach $\mathrm{PyPy}$ sTM's performance level (fannkuch $\star$, nucleotide, mersenne $\star$, parsible). One possible explanation is that JyTHON scales better than PyPy-sTM with the number of available threads and thereby makes up for the disadvantage in sequential performance. Hence, we now continue with the evaluation of the scalability of the vMs.

To evaluate the scalability of PyPy-stM, we must consider an important parameter for PyPy-STM's runtime. The World limit is a parameter that limits the number of transactions that can execute in parallel. As a result, the parameter also limits the number of threads that can make progress in parallel. This parameter may therefore artificially limit the degree of parallelism that an application can utilize with PyPy-sTM.

In Section 3.3, we argued for an environment with a low number of CPU cores, and we, thus, limited the number of threads $T$ to 8 in the above evaluation. Indeed, PyPy-stm's World limit $W$ also defaults to 8 . Hence, we did not observe the behavior of PyPy-stm when the number of threads exceeds the number of Worlds, i.e., $T>W$. Now, we evaluate the effect of this World limit on the scalability of PyPy-sTM by comparing configurations of PyPy-STM with $W$ set to 4 , 8,12 , and 16 . These configurations are respectively called PyPy-STM $_{W 4}$, PyPy-stm, PyPy-STM ${ }_{W 12}$, and PyPy-STMW16.

In Figure 5, we compare the scalability of JYTHON, IRONPython, and the above configurations of PyPy-stm. Since $\mathrm{PyPy}$ does not scale, we exclude it from the figure. The figure shows how well a program on a vM scales with the number of threads (up to $T=16$ ). The metric is the speedup of an execution compared to the single-threaded execution on the same vM, i.e., the execution time of the single-threaded execution divided by the measured execution time.

Under the assumption of perfect scalability, for configurations of PyPy-STM, we expect the maximum speedup to be reached at the point of $T=W$, i.e., when the number of threads matches the number of Worlds. For $T<W$, a benchmark does not exploit all available parallelism, and for $T>W$, the benchmark's threads may have to compete for Worlds. JyTHON and IRONPython are not bound by a World limit and should therefore display the maximum speedup at the maximum number of available threads, i.e., at $T=16$.

JyтноN is highly scalable in 6 out of the 7 benchmarks

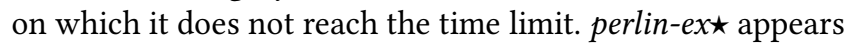
to be an exception. JyThon scales better than all configurations of PyPy-sтм in nucleotide, mersenne $\star$, regex-dna $\star$, and mandelbrot $\star$. Especially in the first two benchmarks, JYTHON's 8-threading performance recovered exceptionally well compared to PyPy-sтм (see Figure 4), which supports the explanation that JyTHON's scalability can make up for the relative disadvantage in sequential performance.

JутноN shows that the benchmarks fannkuch $\star$, nucleotide, mandelbrot $\star$, mersenne $\star$, and regex-dna $a$ allow for more parallelism than can be extracted with 8 threads, i.e., more than 8 threads helped to further reduce the execution time. PyPy$\mathrm{STM}_{\mathrm{W} 16}$ shows that the same is true for richards $\star$. In contrast, the benchmarks nqueens $\star$, parsible, perlin-ex $\star$, and raytrace $\star$ appear to have limited parallelism. Beyond $T=10$, no vM shows a significant improvement in execution time. Limiting factors for a benchmark's parallelism are a limited amount of work to distribute onto threads and overhead from thread management, which cancels out the parallelization benefits.

A particular behavior is shown by fannkuch $\star$, which shows no scalability between 8 and 15 threads, but then sharply improves on 16 threads. The cause for this behavior is a load imbalance within the benchmark, which internally distributes the workload onto 16 tasks. As a result, the benchmark's execution time is dominated by threads that execute 2 tasks while the other threads are idling after completing 1 task. The load is balanced again at $T=16$, at which point all threads execute just 1 task.

Compared to Jython, IronPython's scalability is lower, except in regex-dna , where both vms scale identically, and

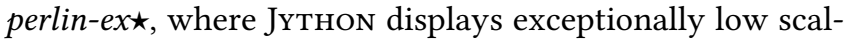
ability. IRonPython's scalability is also generally behind PyPy-stm's and often stagnates or degrades with more than 6 threads. Hence, IronPython cannot make up for its medium sequential performance with scalability. Often, IronPython's scalability barely exceeds that of PyPy-sTM ${ }_{W 4}$, which cannot scale beyond 4 threads due to its low World limit.

Comparing the configurations of PyPy-STM, the different World limits become especially apparent in mandelbrot $\star$, mersenne $\star$, regex-dna $\star$, and richards $\star$. In these benchmarks, PyPy-stm shows better scalability than PyPy-STM ${ }_{W 4}$, PyPySTM 12 better than PyPy-STM, and PyPy-STMW16 better than PyPY-STM ${ }_{W 12}$. Each vM scales up to its respective World limit. When the number of threads starts to exceed the World limit, the performance degrades continuously. The performance degradation is expected since distributing the same amount of work onto more and more threads increases the overhead of starting, managing, and synchronizing these threads. Additionally, threads that must wait for a World to become available are put to sleep, and waking them up when the World becomes available takes extra time. The behavior with $T>W$ is similar to the non-scalability of the GIL.

A parameter that limits the amount of parallelism, such as this World limit of PyPy-STM, can limit the performance of a parallel vм if enough parallelism is available in the program and in the host hardware. Hence, Jython and IronPython, which do not require adjusting such a parameter, have an advantage. Further, the scalability of JyTHON tends to be superior to PyPY-STM, even when choosing a high World limit for the latter. Hence, even if the results are inconclusive, 

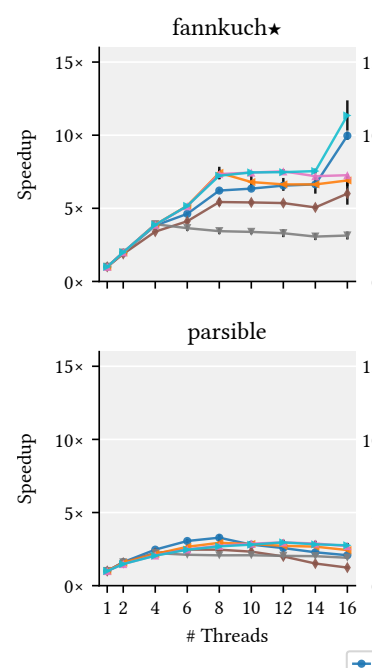

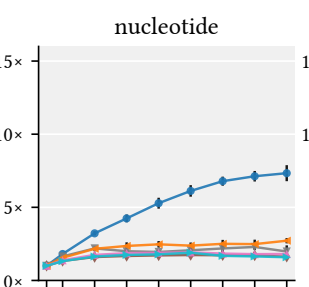

perlin-ex
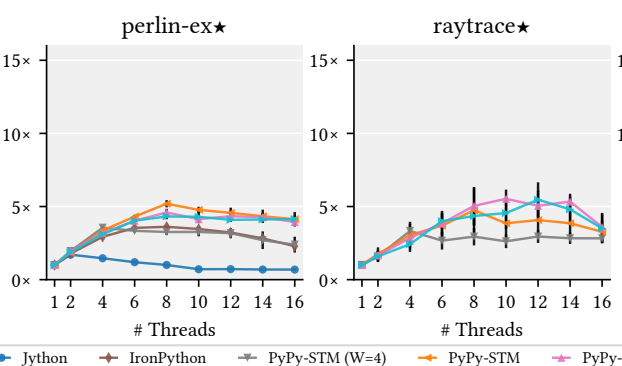

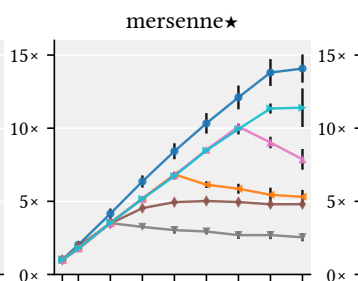

regex-dna $\star$

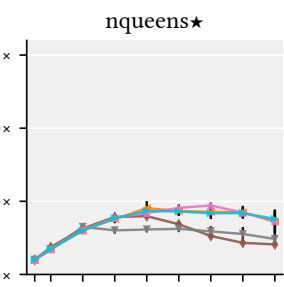

richards $\star$

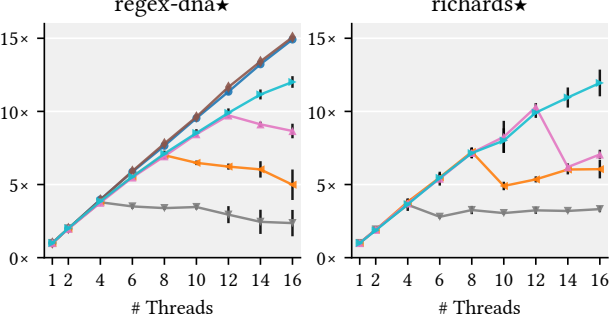

Figure 5. Scalability of vms with the number of threads available. The baseline for each vm is the vm's single-threading performance. Values greater than 1 signify a shorter, and therefore better, execution time.

we identify a tendency for better scalability for high thread counts in parallel vms that use the fine-grained locking approach. This better scalability can make up for the lower sequential performance compared to PyPy-STM, a parallel vM that instead relies on software transactional memory.

\subsection{Best-Performing Configurations}

The scalability of a VM is an important factor to reach high performance. However, the scalability can only partially make up for a low sequential performance level. In the end, the absolute execution times are the determining factor of a vM's performance. In Table 4, we report the lowest execution times that were measured for each benchmark and on each vM. The times are annotated with the number of threads with which they were measured.

For the majority of benchmarks, JYTHON achieves its fastest execution times at $T=16$, i.e., JyTHON scales up to 16 threads. For nucleotide, this scalability leads to the overall shortest execution time as measured on any vM. parsible and perlin$e x \star$ display a different behavior: JутноN's scaling ends at $T=8$ and $T=2$, and execution times get longer after that point. Hence, parsible's and perlin-ex»'s shortest execution times were measured at those thread counts. For parsible, this time turns out to be the overall shortest execution time achieved by any of the compared vms.

IRONPython shows mixed results. Only 2 out of 8 benchmarks achieved the best result at $T=16$. Again, these results show that IronPython's scalability is behind that of JyThON and PyPy-stm. In no case does IronPython have the shortest execution time of all vms.

In a majority of benchmarks, PyPy-STM $\mathrm{W}_{16}$ achieves the overall best performance. The high World limit of $W=16$ allows programs to scale up to $T=16$, and the STM appears to make effective use of hardware resources. The exceptions are nucleotide, nqueens $\star$, parsible, and perlin-ex $\star$, which run faster with a lower World limit. These cases show that increasing the World limit can have drawbacks. With more Worlds, PyPy-sTM must manage more memory. Even if not all Worlds are active, i.e., the number of active threads is smaller than the number of Worlds, not all overhead due to the additional Worlds is avoided. Hence, for applications with limited available parallelism, the choice of $W=8 \mathrm{can}$ yield higher performance than $W=16$. How to choose the best value for $W$ without measuring the result is unclear.

\section{Related Work}

The language Ruby is in a similar situation as Python. The reference implementation of Ruby also uses a GIL to synchronize the vM under concurrent execution, thereby preventing parallel execution. Indeed, mirroring the ecosystem of parallel Python vms, JRuby [5] and IronRuby [4] are the Ruby equivalents to JyTHON and IrONPython. Both are parallel vMs for Ruby, and both use the fine-grained locking approach. Hence, Ruby's situation is comparable to Python's, and similar trade-offs can be made.

Instead of using software transactional memory or finegrained locking to avoid the GIL, attempts were made to use hardware transactional memory instead [25, 30, 35]. So far, these proof of concepts did not result in a complete and unrestricted parallel vm for either Ruby or Python.

The general topic of introducing parallel execution in dynamic language vMs is being addressed by slow-path barricading [34], the development of a thread-safe object model [11], and low-overhead, synchronized collections [12]. These approaches address only a subset of Python's concurrency semantics, omitting sc in particular. 
Table 4. Mean execution times of benchmark iterations in seconds (standard deviations below $5 \%$ are omitted), additionally annotated with the number of threads $T$ on which the time was achieved. For each benchmark, the best result is highlighted.

\begin{tabular}{|c|c|c|c|c|c|c|}
\hline Benchmarks & JYTHON & IRONPYTHON & PyPy-STMW4 & PyPy-stm & PyPy-STMW12 & PyPy-STMW16 \\
\hline fannkuch $\star$ & $6.03 \begin{array}{l}T=16 \\
\pm 0.2\end{array}$ & $\begin{array}{cl}44.39 & T=16 \\
& \pm 6.3\end{array}$ & $11.87 \begin{array}{l}T=4 \\
\pm 0.1\end{array}$ & $6.28 \begin{array}{l}T=8 \\
\pm 0.4\end{array}$ & $6.21 \begin{array}{c}T=12 \\
\pm 0.1\end{array}$ & $\begin{array}{c}4.15 \begin{array}{l}T=16 \\
\pm 0.4\end{array} \\
\end{array}$ \\
\hline nucleotide & $1.87 \begin{array}{l}T=16 \\
\pm 0.1\end{array}$ & $13.05 \begin{array}{l}T=10 \\
\pm 0.5\end{array}$ & $4.10^{T=14} \begin{array}{l} \pm 0.4 \\
\pm 0\end{array}$ & $3.50^{T=16} \begin{array}{l}T=2 \\
\pm 0.2\end{array}$ & $6.02 \begin{array}{l}T=10 \\
\pm 0.7\end{array}$ & $6.40 \begin{array}{l}T=10 \\
\pm 0.6\end{array}$ \\
\hline mandelbrot $\star$ & $16.48 \begin{array}{l}T=16 \\
\pm 0.1\end{array}$ & $11.54 \begin{array}{l}T=10 \\
\pm 0.4\end{array}$ & $2.66^{T=6} \begin{array}{l}T 0.2 \\
\end{array}$ & $1.30^{T=8}$ & $1.01 \begin{array}{c}T=12 \\
\pm 0.1\end{array}$ & $0.94^{T=12}$ \\
\hline mersenne $\star$ & $4.41 \begin{array}{c}T=16 \\
\pm 0.1\end{array}$ & $9.45 \begin{array}{l}T=10 \\
\pm 0.2\end{array}$ & $11.82 \begin{array}{l}T=4 \\
\pm 0.2\end{array}$ & $6.07 \begin{array}{c}T=8 \\
\pm 0.2\end{array}$ & $4.12 \begin{array}{l}T=12 \\
\pm 0.1\end{array}$ & $3.67 \begin{array}{l}T=14 \\
\pm 0.1\end{array}$ \\
\hline nqueens $\star$ & - & $2.86^{T=8} \begin{array}{c}T=1 \\
\pm 0.1\end{array}$ & $0.90^{T=4}$ & $0.65^{T=8} \begin{array}{c}T=1 \\
\pm 0.1\end{array}$ & $0.63^{T=12}$ & $0.69^{T=8}$ \\
\hline parsible & $2.46 \begin{array}{l}T=8 \\
\pm 0.2\end{array}$ & $4.73 \begin{array}{l}T=6 \\
\pm 0.1\end{array}$ & $3.81 \begin{array}{l}T=4 \\
\pm 0.1\end{array}$ & $3.08 \begin{array}{l}T=8 \\
\pm 0.1\end{array}$ & $3.06 \begin{array}{l}T=12 \\
\pm 0.2\end{array}$ & $3.17 \begin{array}{l}T=12 \\
\pm 0.1\end{array}$ \\
\hline perlin-ex $\star$ & $49.15^{T=2} \begin{array}{l}T=2 \\
\pm 0.4\end{array}$ & $24.82 \begin{array}{l}T=8 \\
\pm 0.3\end{array}$ & $4.51 \begin{array}{l}T=4 \\
\pm 0.2\end{array}$ & $3.10 \begin{array}{c}T=8 \\
\pm 0.1\end{array}$ & $3.51 \begin{array}{l}T=8 \\
\pm 0.2\end{array}$ & $3.74 \begin{array}{l}T=8 \\
\pm 0.2\end{array}$ \\
\hline raytrace $\star$ & - & - & $5.49 \begin{array}{l}T=4 \\
\pm 0.7\end{array}$ & $4.26 \begin{array}{c}T=8 \\
\pm 1.2\end{array}$ & $3.37 \begin{array}{c}T=10 \\
\pm 0.3\end{array}$ & $3.46 \begin{array}{l}T=12 \\
\pm 1.1\end{array}$ \\
\hline regex-dna $\star$ & $5.11^{T=16}$ & $15.25^{T=16}$ & $3.04 \begin{array}{l}T=4 \\
\pm 0.1\end{array}$ & $1.64^{T=8}$ & $1.18^{T=12}$ & $0.97^{T=16}$ \\
\hline richards $\star$ & - & - & $9.66 \begin{array}{l}T=4 \\
\pm 1.4\end{array}$ & $4.81 \begin{array}{l}T=8 \\
\pm 0.2\end{array}$ & $3.37 \begin{array}{l}T=12 \\
\pm 0.1\end{array}$ & $2.91 \begin{array}{c}T=16 \\
\pm 0.2\end{array}$ \\
\hline
\end{tabular}

Parallelism can also be exploited with models that are different from the multithreading, shared-memory model. For example, through automatic parallelization [19, 24, 26-28, 36] of loop-based code. This approach works by identifying opportunities for parallelism in sequential code, which can yield benefits that are orthogonal to those yielded by parallel execution through multithreading. Further approaches [10, 13, 23, 31, 32] depend on multiprocessing via task-based parallel programming models, usually depending on message passing instead of shared memory.

\section{Conclusions}

The three parallel Python vms evaluated here differ in their implementation of Python's concurrency semantics. One reason is the approach taken to emulate the semantics. Finegrained locking, employed by Jython and IronPyтноN, has a higher implementation complexity than software transactional memory (STM), which implements sequential consistency (sc) and atomic operations (AO) correctly by design. JyTHON's incomplete semantics and the problematic corner case for IRONPYTHON are evidence for the implementation difficulties caused by fine-grained locking.

The performance levels of JyTHON and IRONPyTHON are around СРутHON's level for sequential performance, and approximately $3 \times$ faster for parallel performance. In contrast, the STM-based PyPy-STM reaches speedups of $3.7 \times$ and $13 \times$ respectively. The exact cause(s) for the different performance levels cannot be established based on the available measurements. The architectural differences between the various vMs are large and may contribute to the performance differences. JythoN and IrONPyTHON both execute on managed language VMs (JVM or CLR), and their JIT compilers are not necessarily well-suited for compiling dynamic languages. In contrast, PyPy-STM's JiT compiler is derived from PyPy's and is optimized for the dynamic language use case. However, the results indicate that the behavior on certain programs coincides with the approach used to emulate Python's concurrency semantics in the parallel vm under test. Future research should explore further aspects of this connection.

Due to their common ancestry, the comparison of PyPySTM with PyPy allows for an estimation of the overhead induced by the sTM approach. The performance of PyPy-STM on these benchmarks is 30\% lower than PyPy's. Hence, using PyPy-stm to run sequential code is almost always slower than using PyPy. Compared to CPython, however, PyPySTM is still $3.7 \times$ faster. Therefore, PyPy-STM goes beyond PyPy and offers high sequential performance approaching that of PyPy, and currently the highest average performance when it comes to parallel execution.

Regarding scalability, vMs following the fine-grained locking approach tend to scale better than the stM-based PyPystm. In some cases, however, PyPy-STM scales better than both Jython and IronPython. Again, singling out the exact reason is difficult due to the architectural differences. The data presents initial evidence for such a connection, and the connection should be examined further in future research.

In summary, parallel vMs for Python can increase an application's performance on modern parallel hardware. The fine-grained locking and the STM approaches show their strengths in different benchmarks. Currently, PyPy-sтм has the best sequential performance among parallel vms. Regarding parallel performance, the slightly better scalability of JyTHON manages to compensate for the lower sequential performance only in a few benchmarks. As a result, PyPy-STM presents the best mix of sequential and parallel performance for multithreaded Python applications and provides an interesting use case of STM in the context of dynamic language implementation.

\section{Acknowledgments}

We thank the reviewers for the excellent feedback, which helped us to significantly improve the paper. Also, we thank the developers of IronPython, Jython, PyPy, and PyPy-stm for advancing the state of parallel Python and for making their work available. 


\section{References}

[1] [n.d.]. CPython. https://www.python.org/.

[2] [n.d.]. A Dynamic Language Runtime (DLR) - Jim Hugunin's Thinking Dynamic. https://blogs.msdn.microsoft.com/hugunin/2007/04/30/adynamic-language-runtime-dlr/.

[3] [n.d.]. IronPython. http://ironpython.net/.

[4] [n.d.]. IronRuby. http://ironruby.net/.

[5] [n.d.]. JRuby. http://jruby.org/.

[6] [n.d.]. Jython. http://www.jython.org/.

[7] [n.d.]. PyPy. http://www.pypy.org/.

[8] 2019. PyPy Benchmarks. https://bitbucket.org/pypy/benchmarks/src/ multithread-runner/.

[9] Edd Barrett, Carl Friedrich Bolz-Tereick, Rebecca Killick, Sarah Mount, and Laurence Tratt. 2017. Virtual Machine Warmup Blows Hot and Cold. Proc. ACM Program. Lang. 1, OOPSLA (Oct. 2017), 52:1-52:27.

[10] Bryan Catanzaro, Michael Garland, and Kurt Keutzer. 2011. Copperhead: Compiling an Embedded Data Parallel Language. In Proceedings of the 16th ACM Symposium on Principles and Practice of Parallel Programming (PPoPP '11). ACM, New York, NY, USA, 47-56.

[11] Benoit Daloze, Stefan Marr, Daniele Bonetta, and Hanspeter Mössenböck. 2016. Efficient and Thread-Safe Objects for Dynamically-Typed Languages. In Proceedings of the 2016 ACM SIGPLAN International Conference on Object-Oriented Programming, Systems, Languages, and Applications (OOPSLA 2016). ACM, New York, NY, USA, 642-659.

[12] Benoit Daloze, Arie Tal, Stefan Marr, Hanspeter Mössenböck, and Erez Petrank. 2018. Parallelization of Dynamic Languages: Synchronizing Built-in Collections. Proc. ACM Program. Lang. 2, OOPSLA (Oct. 2018), 108:1-108:30.

[13] J. J. Galvez, K. Senthil, and L. Kale. 2018. CharmPy: A Python Parallel Programming Model. In 2018 IEEE International Conference on Cluster Computing (CLUSTER). 423-433.

[14] C. A. R. Hoare. 1974. Monitors: An Operating System Structuring Concept. Commun. ACM 17, 10 (Oct. 1974), 549-557.

[15] L. Lamport. 1979. How to Make a Multiprocessor Computer That Correctly Executes Multiprocess Programs. IEEE Trans. Comput. 28, 9 (Sept. 1979), 690-691.

[16] Tobias Lindaaker. 2009. Improving Performance in Jython. http: //journal.thobe.org/2009/07/improving-performance-in-jython.html.

[17] Zoltan Majo, Tobias Hartmann, Marcel Mohler, and Thomas R. Gross. 2017. Integrating Profile Caching into the HotSpot Multi-Tier Compilation System. In Proceedings of the 14th International Conference on Managed Languages and Runtimes (ManLang 2017). ACM, New York, NY, USA, 105-118.

[18] Jeremy Manson, William Pugh, and Sarita V. Adve. 2005. The Java Memory Model. In Proceedings of the 32nd ACM SIGPLAN-SIGACT Symposium on Principles of Programming Languages (POPL '05). ACM, New York, NY, USA, 378-391. 00535.

[19] M. Mehrara, P. Hsu, M. Samadi, and S. Mahlke. 2011. Dynamic Parallelization of JavaScript Applications Using an Ultra-Lightweight Speculation Mechanism. In 2011 IEEE 17th International Symposium on High Performance Computer Architecture. 87-98.

[20] Remigius Meier. 2019. A High-Performance, Parallel Virtual Machine for Python. Doctoral Thesis. ETH Zurich.

[21] Remigius Meier, Armin Rigo, and Thomas R. Gross. 2016. Parallel Virtual Machines with RPython. In Proceedings of the 12th Symposium on Dynamic Languages (DLS 2016). ACM, New York, NY, USA, 48-59.

[22] Remigius Meier, Armin Rigo, and Thomas R. Gross. 2018. Virtual Machine Design for Parallel Dynamic Programming Languages. Proc. ACM Program. Lang. 2, OOPSLA (Oct. 2018), 109:1-109:25.

[23] Stefan C. Müller, Gustavo Alonso, Adam Amara, and André Csillaghy. 2014. Pydron: Semi-Automatic Parallelization for Multi-Core and

the Cloud. In 11th USENIX Symposium on Operating Systems Design and Implementation (OSDI 14). 645-659. https://www.usenix.org/ conference/osdi14/technical-sessions/presentation/muller.

[24] Yeoul Na, Seon Wook Kim, and Youngsun Han. 2016. JavaScript Parallelizing Compiler for Exploiting Parallelism from Data-Parallel HTML5 Applications. ACM Trans. Archit. Code Optim. 12, 4 (Jan. 2016), 64:164:25.

[25] Rei Odaira, Jose G. Castanos, and Hisanobu Tomari. 2014. Eliminating Global Interpreter Locks in Ruby Through Hardware Transactional Memory. In Proceedings of the 19th ACM SIGPLAN Symposium on Principles and Practice of Parallel Programming (PPoPP '14). ACM, New York, NY, USA, 131-142. 00005.

[26] T. Oh, S. R. Beard, N. P. Johnson, S. Popovych, and D. I. August. 2017. A Generalized Framework for Automatic Scripting Language Parallelization. In 2017 26th International Conference on Parallel Architectures and Compilation Techniques (PACT). 356-369.

[27] Richard Plangger and Andreas Krall. 2016. Vectorization in PyPy's Tracing Just-In-Time Compiler. In Proceedings of the 19th International Workshop on Software and Compilers for Embedded Systems (SCOPES '16). ACM, New York, NY, USA, 67-76.

[28] Mohaned Qunaibit, Stefan Brunthaler, Yeoul Na, Stijn Volckaert, and Michael Franz. 2018. Accelerating Dynamically-Typed Languages on Heterogeneous Platforms Using Guards Optimization. In 32nd European Conference on Object-Oriented Programming (ECOOP 2018) (Leibniz International Proceedings in Informatics (LIPIcs)), Todd Millstein (Ed.), Vol. 109. Schloss Dagstuhl-Leibniz-Zentrum fuer Informatik, Dagstuhl, Germany, 16:1-16:29.

[29] Armin Rigo and Samuele Pedroni. 2006. PyPy's Approach to Virtual Machine Construction. In Companion to the 21st ACM SIGPLAN Symposium on Object-Oriented Programming Systems, Languages, and Applications (OOPSLA '06). ACM, New York, NY, USA, 944-953.

[30] Nicholas Riley and Craig Zilles. 2006. Hardware Transactional Memory Support for Lightweight Dynamic Language Evolution. In Companion to the 21st ACM SIGPLAN Symposium on Object-Oriented Programming Systems, Languages, and Applications (OOPSLA '06). ACM, New York, NY, USA, 998-1008. 00013.

[31] Matthew Rocklin. 2015. Dask: Parallel Computation with Blocked Algorithms and Task Scheduling. In Proceedings of the 14th Python in Science Conference. 130 - 136.

[32] Alex Rubinsteyn, Eric Hielscher, Nathaniel Weinman, and Dennis Shasha. 2012. Parakeet: A Just-In-Time Parallel Accelerator for Python. In Presented as Part of the 4th USENIX Workshop on Hot Topics in Parallelism. https://www.usenix.org/conference/hotpar12/workshopprogram/presentation/rubinsteyn.

[33] Kenneth Russell and David Detlefs. 2006. Eliminating SynchronizationRelated Atomic Operations with Biased Locking and Bulk Rebiasing. In Proceedings of the 21st Annual ACM SIGPLAN Conference on ObjectOriented Programming Systems, Languages, and Applications (OOPSLA '06). ACM, New York, NY, USA, 263-272.

[34] James Swaine, Kevin Tew, Peter Dinda, Robert Bruce Findler, and Matthew Flatt. 2010. Back to the Futures: Incremental Parallelization of Existing Sequential Runtime Systems. In Proceedings of the ACM International Conference on Object Oriented Programming Systems Languages and Applications (OOPSLA '10). ACM, New York, NY, USA, 583-597.

[35] Fuad Tabba. 2010. Adding Concurrency in Python Using a Commercial Processor's Hardware Transactional Memory Support. SIGARCH Comput. Archit. News 38, 5 (April 2010), 12-19. 00007.

[36] J. Talbot, Z. DeVito, and P. Hanrahan. 2012. Riposte: A Trace-Driven Compiler and Parallel VM for Vector Code in R. In 2012 21st International Conference on Parallel Architectures and Compilation Techniques (PACT). 43-51. 